\title{
COMPLEX PERSPECTIVE FOR THE PROJECTIVE HEAT MAP ACTING ON PENTAGONS
}

\author{
SCOTT R. KASCHNER AND ROLAND K. W. ROEDER
}

\begin{abstract}
We place Schwartz's work on the real dynamics of the projective heat map $H$ into the complex perspective by computing its first dynamical degree and gleaning some corollaries about the dynamics of $H$.
\end{abstract}

\section{INTRODUCTION}

Let $\mathcal{P}_{N}$ denote the space of projective equivalence classes of $N$-gons in $\mathbb{R P}^{2}$. The projective heat map is a self-mapping of $\mathcal{P}_{N}$ that was introduced by R. Schwartz in the monograph [19]. Suppose $A B, B C$, and $C D$ are three consecutive edges of a polygon $P$. The projective midpoint of $B C$ (with respect to the polygon $P$ ) is defined as $S:=\overleftrightarrow{B C} \cap \overleftrightarrow{Q R}$, where $Q=\overleftrightarrow{A B} \cap \overleftrightarrow{C D}$ and $R=\overleftrightarrow{A C} \cap \overleftrightarrow{B D}$

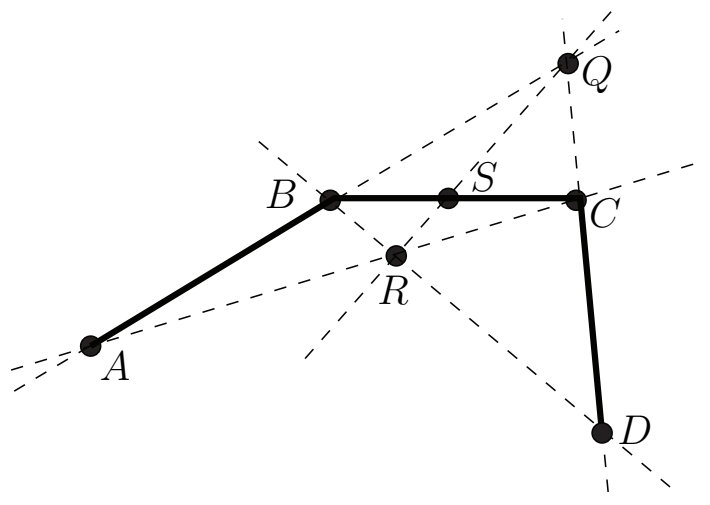

Figure $1 . S$ is the projective midpoint of $B C$.

The projective midpoint of $B C$ typically does not coincide with the Euclidean midpoint of $B C$, however the construction is invariant under projective transformations. Remark also that the projective midpoint may not be defined for certain degenerate configurations of $A, B, C$, and $D$.

For any $N$-gon $P$ let $H(P)$ be the $N$-gon whose vertices are the projective midpoints of the edges of $P$. Because the construction is invariant under projective transformations, $H$ descends to a mapping $H: \mathcal{P}_{N} \rightarrow \mathcal{P}_{N}$ called the projective heat map. (We used a broken arrow to denote that $H$ may not be defined at certain

Received by the editors October 6, 2016, and, in revised form, March 3, 2017.

2010 Mathematics Subject Classification. Primary 37F99; Secondary 32H50.

Key words and phrases. Pullback on cohomology, dynamical degrees. 
polygons for which a sequence of four consecutive vertices $A, B, C$, and $D$ are not in general position.)

In the case of pentagons, the space of projective equivalence classes of polygons $\mathcal{P}_{5}$ is parameterized by a pair of real numbers $(x, y)$ called the flag invariants of the (equivalence class) of the polygon; see [19, Section 3.6]. In these flag coordinates, the projective heat mapping becomes a rational mapping $H: \mathbb{R}^{2} \rightarrow \mathbb{R}^{2}$ given by $\left(x^{\prime}, y^{\prime}\right)=H(x, y)$, where

$$
\begin{aligned}
x^{\prime} & =\frac{\left(x y^{2}+2 x y-3\right)\left(x^{2} y^{2}-6 x y-x+6\right)}{\left(x y^{2}+4 x y+x-y-5\right)\left(x^{2} y^{2}-6 x y-y+6\right)}, \quad \text { and } \\
y^{\prime} & =\frac{\left(x^{2} y+2 x y-3\right)\left(x^{2} y^{2}-6 x y-y+6\right)}{\left(x^{2} y+4 x y-x+y-5\right)\left(x^{2} y^{2}-6 x y-x+6\right)} .
\end{aligned}
$$

This mapping has an obvious symmetry under the reflection $R(x, y)=(y, x)$. It also has a less obvious symmetry under an action of the dihedral group $D_{5}$, corresponding to relabeling the vertices of the pentagon under rotation and reflections. Expressed as a group of birational mappings of the flag coordinates $(x, y) \in \mathbb{R}^{2}$ this action of $D_{5}$ is called the Gauss Group $\Gamma$; see [19, Section 3.8].

In the monograph 19 Schwartz uses computer-assisted proofs to provide a nearly complete description of the dynamics of $H: \mathbb{R}^{2} \rightarrow-\mathbb{R}^{2}$. Highlights of his work include proofs that

(1) Almost any projective equivalence class of a pentagon has orbit under $H$ converging to the class of the regular pentagon [19, Corollary 1.7]. (In flag coordinates, this attracting fixed point is represented by $\left(\phi^{-1}, \phi^{-1}\right)$, where $\phi$ is the golden ratio.)

(2) There is a repelling invariant Cantor set $\mathcal{J C}$ for $H$ on which the dynamics of $H$ is conjugate to the one-sided shift $\sigma: \Sigma_{6} \rightarrow \Sigma_{6}$ on six symbols 19 , Theorem 1.5].

(3) $H$ does not have an invariant rational fibration, i.e., there is no nontrivial pair $(h, f)$ of rational functions $f: \mathbb{R}^{2} \rightarrow \mathbb{R}$ and $h: \mathbb{R} \rightarrow \mathbb{R}$ such that $f \circ H=h \circ f$ [19, Section 15.7]. This can be interpreted as saying the dynamics of $H$ are truly two-dimensional.

Moreover, much of Schwartz's work is dedicated to giving a topological description of the "Julia set" of $H$ defined by $\mathcal{J}:=\mathbb{R}^{2} \backslash \mathcal{W}^{s}\left(\left(\phi^{-1}, \phi^{-1}\right)\right)$. A computer generated image of this Julia set is shown in Figure 2

Schwartz often extends $H$ to the compactification $\mathbb{R P}^{1} \times \mathbb{R} \mathbb{P}^{1}$ and sometimes to the surface $S_{\mathbb{R}}$ obtained by blowing up $\mathbb{R P}^{1} \times \mathbb{R} \mathbb{P}^{1}$ at the three points $(1,1),(\infty, 0)$, and $(0, \infty)$. The surface $S_{\mathbb{R}}$ is well adapted to the symmetries of $H$ under the Gauss Group $\Gamma$, since $\Gamma$ acts on $S_{\mathbb{R}}$ by diffeomorphisms; see [19, Section 10.5].

The formula for $H$ naturally extends to a rational map

$$
H: \mathbb{C P}^{1} \times \mathbb{C P}^{1} \rightarrow \mathbb{C P}^{1} \times \mathbb{C P}^{1}
$$

or even as a rational self-map $H: S \rightarrow S$, where $S$ is the blow-up of $\mathbb{C P}^{1} \times \mathbb{C P}^{1}$ at $(1,1),(\infty, 0)$, and $(0, \infty)$. Starting in the 1990s, Briend-Duval, Fornaess-Sibony, Hubbard-Oberst-Vorth, Hubbard-Papadapol, Ueda, and many others used powerful tools from complex analysis and algebraic geometry to prove strong results about the dynamics of such mappings. The purpose of this note is to place Schwartz's work on the real dynamics of the projective heat mapping $H$ into this complex perspective and to glean some corollaries both about the dynamics of $H$. 


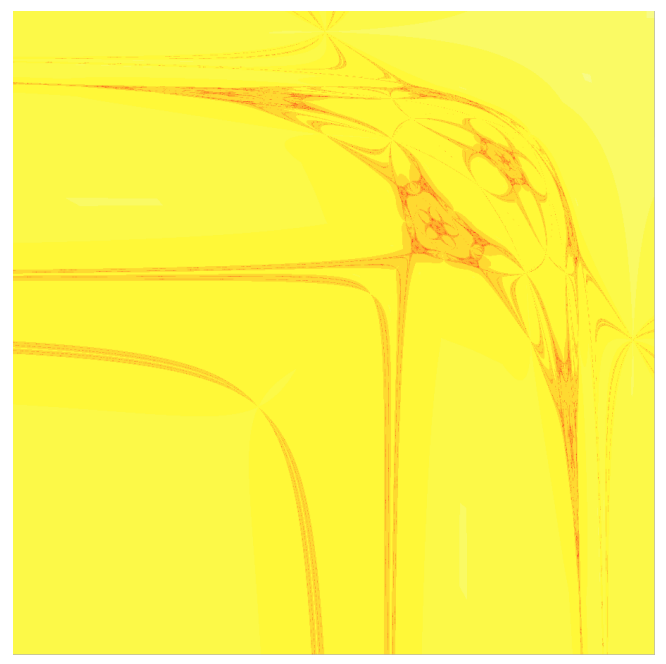

Figure 2. The region $(x, y) \in\left[-9, \phi^{-1}\right]^{2}$, where $\phi$ is the golden ratio. Points in the basin of attraction $\mathcal{W}^{s}\left(\left(\phi^{-1}, \phi^{-1}\right)\right)$ of the attracting fixed point $\left(\phi^{-1}, \phi^{-1}\right)$ are colored yellow. The "Julia Set" $\mathcal{J}:=\mathbb{R}^{2} \backslash \mathcal{W}^{s}\left(\left(\phi^{-1}, \phi^{-1}\right)\right)$ is visible in red.

The coarsest invariants of the dynamics of a rational self-mapping $f: X \rightarrow X$ of a (complex) projective surface $X$ are the dynamical degrees $\lambda_{1}(f)$ and $\lambda_{2}(f)$. The first dynamical degree $\lambda_{1}(f)$ is defined by

$$
\lambda_{1}(f):=\lim _{n \rightarrow \infty}\left\|\left(f^{n}\right)^{*}: \mathrm{H}^{1,1}(X ; \mathbb{C}) \rightarrow \mathrm{H}^{1,1}(X ; \mathbb{C})\right\|^{1 / n},
$$

where $\mathrm{H}^{1,1}(X ; \mathbb{C})$ denotes the Dolbeault cohomology of bidegree $(1,1)$. (In the case considered in this note, the reader can replace it with singular cohomology $\mathrm{H}^{2}(X ; \mathbb{C})$.) There are many subtleties to this definition, including the fact that the rational map $f$ may not be continuous at a finite set of indeterminate points. Nevertheless, there is still a well-defined notion of pullback on cohomology. The caveat is that, unlike for continuous mappings, this pullback may not be functorialone may have $\left(f^{n}\right)^{*} \neq\left(f^{*}\right)^{n}$. This often makes calculation of $\lambda_{1}(f)$ rather technical.

Since we are working in complex dimension two, the second dynamical degree is much simpler: $\lambda_{2}(f)=\operatorname{deg}_{\text {top }}(f)$ is the number of preimages of a generic point under $f$. We refer the reader to [2, 5, 10, 11, 13, 18, for further background on dynamical degrees. The facts we will need about them will be summarized in Section 2 .

The two cases $\lambda_{2}(f)>\lambda_{1}(f)$ and $\lambda_{1}(f)>\lambda_{2}(f)$ correspond to quite different types of dynamics. Building on work of Briend-Duval [3, 4, Guedj and DinhTruong-Nguyen [9, 15] proved that the case $\lambda_{2}(f)>\lambda_{1}(f)$ corresponds to "predominantly repelling dynamics". Meanwhile, building from work of Bedford-LyubichSmillie, Diller-Dujardin-Guedj [6] proved that the case $\lambda_{1}(f)>\lambda_{2}(f)$ corresponds to "predominantly saddle-type dynamics". We refer the reader to the cited papers for details. 
It was proved by Schwartz [19, Section 8] that $\lambda_{2}(H)=6$. Our main technical result is

Theorem 1.1. $\lambda_{1}(H)=4$.

Therefore, the projective heat map $H$ has "large topological degree" and the work of Guedj and Dinh-Truong-Nguyen [9, 15 implies that $H: \mathbb{C P}^{1} \times \mathbb{C P}^{1} \rightarrow \mathbb{C P}^{1} \times \mathbb{C P}^{1}$ has a unique measure $\mu$ of maximal entropy, whose entropy is equal to

$$
\log \lambda_{2}(H)=\log 6 .
$$

It also provides a lower bound on the Lyapunov exponents $\chi_{1,2}$ of $\mu$ by

$$
\chi_{1,2} \geq \frac{1}{2} \log \frac{\lambda_{2}(H)}{\lambda_{1}(H)}=\frac{1}{2} \log \frac{3}{2} .
$$

Meanwhile, the Bernoulli measure on $\Sigma_{6}$ is an invariant measure of entropy $\log 6$ for the one-sided full shift on six symbols $\sigma: \Sigma_{6} \rightarrow \Sigma_{6}$. Pulling it back under the conjugacy between $H: \mathcal{J C} \rightarrow \mathcal{J C}$ and $\sigma: \Sigma_{6} \rightarrow \Sigma_{6}$ results in an invariant measure $\beta$ for $H: \mathcal{J C} \rightarrow \mathcal{J C}$ which also has entropy $\log 6$. We will call $\beta$ the "Bernoulli measure on $\mathcal{J C} "$. We conclude that:

Corollary 1.2. The Bernoulli measure $\beta$ on $\mathcal{J C} \subset \mathbb{R} \mathbb{P}^{1} \times \mathbb{R} \mathbb{P}^{1}$ is the unique measure of maximal entropy for $H: \mathbb{C P}^{1} \times \mathbb{C P}^{1} \rightarrow \mathbb{C P}^{1} \times \mathbb{C P}^{1}$. Its Lyapunov exponents are bounded from below by $\frac{1}{2} \log \frac{3}{2}$.

The dynamical degrees of rational maps having an invariant fibration were studied by Dinh-Nguyen and Dinh-Nguyen-Truong in 7, 8. Their work implies that if a rational self-map of a surface has an invariant fibration, then $\lambda_{1}(f)$ divides $\lambda_{2}(f)$; see Lemma 2.4, below. We therefore also conclude that:

Corollary 1.3. The projective heat map $H: \mathbb{C P}^{1} \times \mathbb{C P}^{1} \rightarrow \mathbb{C P}^{1} \times \mathbb{C P}^{1}$ has no invariant fibration.

If $H$ preserved a real fibration $(f, g)$, their complexifications would serve as an invariant complex fibration for $H$. In particular, $H: \mathbb{R P}^{1} \times \mathbb{R P}^{1} \rightarrow \mathbb{R P}^{1} \times \mathbb{R} \mathbb{P}^{1}$ does not have an invariant fibration, thus giving a cohomological re-proof of one of Schwartz's results.

Remark 1.4. Computation of the first dynamical degree of a planar rational map is relatively standard for specialists in several variable complex dynamics. However, the purpose of this note is to relate the dynamical consequences of this calculation to Schwartz's work. In order to make this paper more accessible to readers outside the field of several variables complex dynamics, we have intentionally included many details that otherwise might have been omitted.

Section 2 contains background material on dynamical degrees for rational maps of surfaces and how they can be used to rule out invariant fibrations. Sections 3 and 4 are devoted to the calculation that $\lambda_{1}(H)=4$, i.e., to proving Theorem 1.1 . There are many interesting further questions about the complex dynamics of the projective heat map. Therefore, we conclude in Section 5 by describing a couple of interesting directions for further study. 


\section{BACKGROUND ON DYNAMICAL DEGREES AND ALGEBRAIC STABILITY}

In this section we will give the background needed for computing $\lambda_{1}(H)$. Let us focus on a somewhat restricted context suitable for this paper. Suppose $X$ and $Y$ are complex projective algebraic surfaces (e.g. a blow-up of $\mathbb{C P}^{1} \times \mathbb{C P}^{1}$ at finitely many points) and $f: X \rightarrow Y$ is a rational map. We denote the indeterminacy locus of $f$ by $I(f)$, which is a finite set of points. Throughout the section we will suppose $f$ is dominant, meaning that $f(X \backslash I(f))$ is not contained in an algebraic hypersurface of $Y$.

2.1. Pullback on cohomology. It is a well-known fact [20, Ch. IV, §3.3] there is a finite sequence of blow-ups $\pi: \widetilde{X} \rightarrow X$ so that $f$ lifts to a holomorphic map $\widetilde{f}: \widetilde{X} \rightarrow Y$, making the following diagram commute:

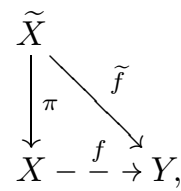

wherever $f \circ \pi$ is defined. Each of these blow-ups is done over a point of $I(f)$.

One uses (2.1) to define $f^{*}: \mathrm{H}^{1,1}(Y ; \mathbb{C}) \rightarrow \mathrm{H}^{1,1}(X ; \mathbb{C})$ by

$$
f^{*}(\alpha):=\pi_{*}\left(\widetilde{f}^{*} \alpha\right)
$$

for any $\alpha \in \mathrm{H}^{1,1}(Y ; \mathbb{C})$. Here, $\pi_{*}: \mathrm{H}^{1,1}(\widetilde{X} ; \mathbb{C}) \rightarrow \mathrm{H}^{1,1}(X ; \mathbb{C})$ is defined by

$$
\pi_{*}:=\mathrm{PD}_{X}^{-1} \circ \pi_{\#} \circ \mathrm{PD}_{\widetilde{X}}
$$

with $\pi_{\#}$ denoting the push forward on homology and PD denoting the Poincaré duality isomorphism. The definition of the pullback $f^{*}$ is well defined, independent of the choice of resolution of indeterminacy (2.1); see, for example, [17, Lemma 3.1].

If $C \subset X$ is an irreducible algebraic curve, then, since $C$ has real dimension two and is singular at only finitely many points, it has a well-defined fundamental homology class $\{C\}$ and cohomology class $[C]=\mathrm{PD}_{X}^{-1}(\{C\})$. From a more sophisticated point of view, $C$ defines a locally principal divisor $(C)$ and $[C]$ is its Chern class; [14. The definition of $f^{*}$ simplifies in this case:

Lemma 2.1. Let $f: X \rightarrow Y$ be a dominant rational map between two projective surfaces. Suppose $C \subset Y$ is an irreducible algebraic curve. Then,

$$
f^{*}[C]=\sum_{\substack{D \subset f^{-1}(C) \\ \text { irreducible }}} m_{D}[D],
$$

where $f^{-1}(C)=\overline{\left(\left.f\right|_{X \backslash I(f)}\right)^{-1} C}$ and the multiplicity $m_{D}$ is the order of vanishing of $\psi \circ f$ at any smooth point $p \in D \backslash I(f)$, with $\psi$ being a local defining equation for $C$ at $f(p)$ (chosen to vanish to order 1 at smooth points of $C$ ). 
Proof. For a holomorphic map $h: X \rightarrow Y$ the pullback action on locally principal divisors and on cohomology are related by the commutative diagram:

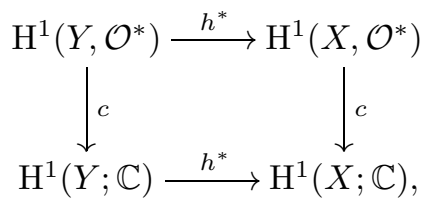

where $\mathcal{O}^{*}$ denotes the sheaf of germs of nonvanishing holomorphic functions and the vertical arrows denote taking the Chern class; see [14, p. 139]. Since (2.3) is a special case of the definition of how one pulls back locally principal divisors, this diagram justifies (2.3) in the case of a holomorphic map.

Now, suppose $f: X \rightarrow Y$ is rational, that we have the resolution of indeterminacy $\tilde{f}: \widetilde{X} \rightarrow Y$ as in (2.1), and let $C \subset Y$ be an irreducible algebraic curve. By the discussion in the previous paragraph, (2.3) applies to $\tilde{f}: \tilde{X} \rightarrow Y$ giving

$$
\tilde{f}^{*}[C]=\sum_{\substack{A \subset \widetilde{f}^{-1}(C) \\ \text { irreducible }}} m_{A}[A]
$$

with $m_{A}$ being the order of vanishing of $\psi \circ \widetilde{f}$ at any smooth point of $A$. Therefore, we have

$$
f^{*}[C]=\pi_{*} \tilde{f}^{*}[C]=\sum_{\substack{A \subset \tilde{f}^{-1}(C) \\ \text { irreducible }}} m_{A} \pi_{*}[A] .
$$

If $A$ is an irreducible component of $\tilde{f}^{-1}(C)$ with $\pi(A)$ a single point, then by the definition of push forward on homology we have $\pi_{*}[A]=0$. Otherwise, $\pi(A)$ is an irreducible algebraic curve in $X$. In this case, $A$ intersects the exceptional divisors of $\pi$ in finitely many points, so that $\pi: A \rightarrow \pi(A)$ is one-to-one away from finitely many points and hence $\pi_{*}[A]=[\pi(A)]$. We conclude that

$$
f^{*}[C]=\sum_{\substack{A \subset \widetilde{f}^{-1}(C) \text { irreducible } \\ \pi(A) \text { not a point }}} m_{A}[\pi(A)] .
$$

Suppose $p$ is any smooth point of $\pi(A) \backslash I(f)$. Then, $\pi$ is a biholomorphic map from a neighborhood $U$ of $\pi^{-1}(p)$ to a neighborhood $V$ of $p$. Therefore, if $\psi$ is a local defining equation for $C$ in a neighborhood of $f(p)=\widetilde{f}\left(\pi^{-1}(p)\right)$ the order of vanishing of $\psi \circ f=\psi \circ \tilde{f} \circ\left(\left.\pi\right|_{U}\right)^{-1}$ at $p$ is the same as that of $\psi \circ \tilde{f}$ at $\pi^{-1}(p)$. This allows us to replace the $m_{A}$ in (2.7) with $m_{\pi(A)}$. We obtain

$$
f^{*}[C]=\sum_{\substack{D \subset \pi\left(\tilde{f}^{-1}(C)\right) \text { irreducible } \\ D \text { not a point }}} m_{D}[D] .
$$

Finally, note that each of the blow-ups done to achieve the resolution of indeterminacy (2.1) occurs over a point of $I(f)$. Therefore,

$$
f^{-1}(C)=\overline{\left(\left.f\right|_{X \backslash I(f)}\right)^{-1} C} \text { and } \pi \circ \tilde{f}^{-1}(C)
$$


differ by at most a subset of the finite set $I(f)$, giving a bijection between irreducible components of $f^{-1}(C)$ and irreducible components of $\pi\left(\tilde{f}^{-1}(C)\right)$ that are not a point. This gives (2.3) from (2.8).

\subsection{Algebraic stability and strategy for computing $\lambda_{1}$.}

Proposition 2.2 (Diller-Favre [5, Thm. 1.14]). Let $f: X \rightarrow X$ by a rational selfmap of a projective surface. Then, $\left(f^{n}\right)^{*}=\left(f^{*}\right)^{n}$ for all $n \geq 1$ if and only if there is no curve $C$ and no iterate $m$ such that $f^{m}\left(C \backslash I_{f^{m}}\right) \subset I_{f}$.

When either of the two equivalent conditions stated in Proposition 2.2 hold, we will call $f: X \rightarrow X$ algebraically stable. (Readers who prefer to see a proof of Proposition 2.2 that is based on algebraic geometry instead of analysis can refer to [17, Prop. 1.4].)

If $C$ is a curve such that $f\left(C \backslash I_{f}\right)$ is a point, we will say that $C$ is collapsed by $f$. Since $I_{f}$ is a finite set of points, the second equivalent condition for algebraic stability (from Proposition 2.2) asserts that there is no curve $C$ that is collapsed by an iterate of $f$ into $I_{f}$. In particular, if $f$ does not collapse any curve, then $f$ is algebraically stable.

Proposition 2.3 (Dinh-Sibony [11, Cor. 7]). Suppose $f: X \rightarrow X$ and $g: Y \rightarrow Y$ are rational maps of projective surfaces that are conjugate by means of a birational map $\pi: X \rightarrow Y$. Then, $\lambda_{1}(f)=\lambda_{1}(g)$.

(Proposition 2.3 actually holds considerably greater generality, including in arbitrary dimensions, but we will only need the simplest form, as stated here.)

Based on these two propositions, there is a clear strategy for computing the first dynamical degree of a rational map $f: X \rightarrow X$. One should try to do a sequence of blow-ups $\pi: \widetilde{X} \rightarrow X$ in order to make the lifted map $\widetilde{f}: \widetilde{X} \rightarrow \widetilde{X}$ satisfy the second equivalent condition from Proposition 2.2, and hence be algebraically stable. Then

$$
\lambda_{1}(f)=\lambda_{1}(\tilde{f})=\text { spectral radius }\left(\tilde{f}^{*}: \mathrm{H}^{1,1}(\tilde{X}) \rightarrow \mathrm{H}^{1,1}(\tilde{X})\right),
$$

where the first equality follows from applying Proposition 2.3 to the conjugacy $\pi$ between $f$ and $\widetilde{f}$, and the second equality follows from Proposition 2.2. Finally, if the cohomology $\mathrm{H}^{1,1}(\widetilde{X})$ is generated by the fundamental classes of algebraic curves, one can use Lemma 2.1 to compute the spectral radius on the right hand side of (2.9).

\subsection{Ruling out invariant fibrations by means of dynamical degrees.}

Lemma 2.4. Suppose $f: X \rightarrow X$ is a dominant rational map of a projective surface that has an invariant fibration. Then, $\lambda_{1}(f)$ divides $\lambda_{2}(f)$.

Proof. Suppose that $Y$ is a one-dimensional projective curve and that $f: X \rightarrow X$ is semi-conjugate to a rational map $g: Y \rightarrow Y$, by a rational mapping $\pi: X \rightarrow Y$. The formula from [7,8] gives that:

$$
\begin{aligned}
& \lambda_{1}(f)=\max \left(\lambda_{1}(g) \lambda_{0}(f \mid \pi), \lambda_{0}(g) \lambda_{1}(f \mid \pi)\right), \quad \text { and } \\
& \lambda_{2}(f)=\lambda_{1}(g) \lambda_{1}(f \mid \pi) .
\end{aligned}
$$


Here, the fiber-wise dynamical degrees are defined as

$$
\lambda_{i}(f \mid \pi):=\lim _{n \rightarrow \infty}\left\|\left(f^{n}\right)^{*}\left(\omega_{X}^{i}\right) \wedge \pi^{*}\left(\omega_{Y}\right)\right\|^{1 / n}
$$

for $i=0,1$, where $\omega_{X}$ and $\omega_{Y}$ are the Fubini-Study forms on $X$ and $Y$ respectively and $\|\cdot\|$ denotes the mass of a current. (The exponent on $\omega_{Y}$ is 1 since $\operatorname{dim}(Y)=1$.) It is always true that the 0 -th dynamical degree of a mapping is 1 , so that $\lambda_{0}(g)=1$. (This is why we didn't mention 0-th dynamical degrees in the introduction.) Meanwhile, it follows immediately from (2.10) that $\lambda_{0}(f \mid \pi)=1$. Therefore, $\lambda_{1}(f)=\max \left(\lambda_{1}(g), \lambda_{1}(f \mid \pi)\right)$. In either case, the result follows from $\lambda_{2}(f)=\lambda_{1}(g) \lambda_{1}(f \mid \pi)$.

Corollary 1.3 follows.

\section{Algebraic stability on a suitable Blow-up of $\mathbb{C P}^{1} \times \mathbb{C P}^{1}$.}

We use multi-homogeneous coordinates $([X: U],[Y: V])$ on $\mathbb{C P}^{1} \times \mathbb{C P}^{1}$, where the affine coordinates from (1.1) correspond to $(x, y)=(X / U, Y / V)$. There are three other "standard" choices of local coordinates on $\mathbb{C P}^{1} \times \mathbb{C P}^{1}$ given by

$$
(u, y):=(U / X, Y / V), \quad(x, v):=(X / U, V / Y), \quad \text { and } \quad(u, v):=(U / X, V / Y) .
$$

In the $(x, y)$ local coordinates, the critical set of $H: \mathbb{C}^{2} \rightarrow \mathbb{C}^{2}$ consists of the following five irreducible curves:

$$
\begin{aligned}
C_{1} & =\{x y-1=0\}, \\
C_{2} & =\{2 x y+x+y-4=0\}, \\
C_{3} & =\left\{x^{2} y^{2}-6 x y-y+6=0\right\}, \\
C_{4} & =\left\{x^{2} y^{2}-6 x y-x+6=0\right\}, \text { and } \\
C_{5} & =\left\{x^{6} y^{6}-10 x^{5} y^{5}-x^{6} y^{3}+2 x^{5} y^{4}+2 x^{4} y^{5}-x^{3} y^{6}-4 x^{5} y^{3}+39 x^{4} y^{4}-4 x^{3} y^{5}+3 x^{5} y^{2}\right. \\
& -12 x^{4} y^{3}-12 x^{3} y^{4}+3 x^{2} y^{5}+10 x^{4} y^{2}-47 x^{3} y^{3}+10 x^{2} y^{4}-3 x^{4} y^{2}+22 x^{3} y^{2}+22 x^{2} y^{3} \\
& \left.-3 x y^{4}-12 x^{3} y-2 x^{2} y^{2}-12 x y^{3}-6 x^{2} y-6 x y^{2}+9 x^{2}+21 x y+9 y^{2}-9 x-9 y=0\right\} .
\end{aligned}
$$

Lemma 3.1. The only curves collapsed by $H: \mathbb{C P}^{1} \times \mathbb{C P}^{1} \rightarrow \mathbb{C P}^{1} \times \mathbb{C P}^{1}$ are $C_{1}, \ldots, C_{4}$.

- $C_{1}$ and $C_{2}$ are collapsed by $H$ to $p_{1}:=\{(x, y)=(1,1)\}$,

- $C_{3}$ is collapsed by $H$ to $p_{2}:=\{(x, y)=(\infty, 0)\}=\{(u, y)=(0,0)\}$, and

- $C_{4}$ is collapsed by $H$ to $p_{3}:=\{(x, y)=(0, \infty)\}=\{(x, v)=(0,0)\}$.

The curve $C_{5}$ is not collapsed by $H$.

Proof. Any curve that is collapsed by $H$ is in the critical set, and it is also easy to verify in $(u, v)$ coordinates that the lines at infinity, $\{u=0\}$ and $\{v=0\}$, are not collapsed. Thus, we need only consider $C_{1}, \ldots, C_{5}$.

The variable $x$ occurs at most linearly in the defining equations for $C_{1}$ and $C_{2}$. One can therefore solve for $x$ as a function of $y$. Substituting into the defining equation for $H$ (1.1) yields that each curve $C_{1}$ and $C_{2}$ collapses to $p_{1}$. 
To see that $C_{3}$ is collapsed to $p_{2}$, we use $(x, y)$ coordinates in the domain and $(u, y)=(1 / x, y)$ in codomain:

$$
\begin{aligned}
u^{\prime}=1 / x^{\prime} & =\frac{\left(x y^{2}+4 x y+x-y-5\right)\left(x^{2} y^{2}-6 x y-y+6\right)}{\left(x y^{2}+2 x y-3\right)\left(x^{2} y^{2}-6 x y-x+6\right)}, \quad \text { and } \\
y^{\prime} & =\frac{\left(x^{2} y+2 x y-3\right)\left(x^{2} y^{2}-6 x y-y+6\right)}{\left(x^{2} y+4 x y-x+y-5\right)\left(x^{2} y^{2}-6 x y-x+6\right)} .
\end{aligned}
$$

Since the defining equation of $C_{3}$ appears in the numerator of both $u^{\prime}$ and $y^{\prime}$, it follows that $C_{3}$ collapses to $(u, y)=(0,0)$. The fact that $C_{4}$ is collapsed by $H$ to $p_{3}$ follows by symmetry under $R$, or by using a similar calculation.

Meanwhile, $(x, y)=(0,0)$ and $(x, y)=(0,1)$ are on $C_{5}$ and they map by $H$ to different points.

Let $\mathcal{X}$ be the blow-up of $\mathbb{C P}^{1} \times \mathbb{C P}^{1}$ at $p_{1}, p_{2}$, and $p_{3}$, let $E_{1}, E_{2}$, and $E_{3}$ be the resulting exceptional divisors, respectively, and let $\pi: \mathcal{X} \rightarrow \mathbb{C P}^{1} \times \mathbb{C P}^{1}$ be the canonical projection. We let $\mathcal{H}: \mathcal{X} \rightarrow \mathcal{X}$ be the lift of $H$ to this space such that the following diagram commutes:

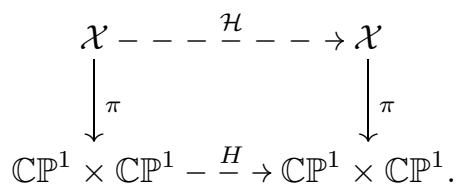

For any algebraic curve $C \subset \mathbb{C P}^{1} \times \mathbb{C P}^{1}$ let

$$
\widetilde{C}=\overline{\pi^{-1}\left(C \backslash\left\{p_{1}, p_{2}, p_{3}\right\}\right)}
$$

denote the proper transform of $C$ under $\pi$.

The local coordinates $(x, y),(u, y),(x, v)$ and $(u, v)$ on $\mathbb{P}^{1} \times \mathbb{P}^{1}$ continue to serve as local coordinates on $\mathcal{X}$ away from the exceptional divisors $E_{1}, E_{2}$, and $E_{3}$. We now set up some standard choices of local coordinates on $\mathcal{X}$ near these exceptional divisors. Consider the local coordinates $(a, b)=(x-1, y-1)$ on $\mathbb{P}^{1} \times \mathbb{P}^{1}$ centered at $p_{1}$. One can describe a neighborhood of $E_{1}$ in $\mathcal{X}$ using two systems of local coordinates. The local coordinates $\left(a, m_{1}\right)=(a, b / a)$ describe all directions of approach to $p_{1}$, except along the line $\{x=1\}$, which corresponds to $m_{1}=\infty$. (We actually won't need the other systems of coordinates in this paper.) Similarly, the local coordinates $\left(u, m_{2}\right)=(u, y / u)$ describe a neighborhood of all but one point of the exceptional divisor $E_{2}$, and the local coordinates $\left(v, m_{3}\right)=(v, x / v)$ describe a neighborhood of all but one point of the exceptional divisor $E_{3}$. See Figure 3 ,

Lemma 3.2. $\mathcal{H}: \mathcal{X} \rightarrow \mathcal{X}$ does not collapse any curves and is therefore algebraically stable. In particular,

- $\mathcal{H}$ maps $\widetilde{C_{1}}$ and $\widetilde{C_{2}}$ onto all of $E_{1}$,

- $\mathcal{H}$ maps $\widetilde{C_{3}}$ onto all of $E_{2}$, and

- $\mathcal{H}$ maps $\widetilde{C_{4}}$ onto all of $E_{3}$.

Proof. If $\mathcal{H}$ collapses a curve $A$ that is not one of the exceptional divisors $E_{i}$ of $\pi$, then $\pi(A)$ is a curve that is collapsed by $H$. Therefore, since $C_{1}, \ldots, C_{4}$ are the only curves collapsed by $H$, it suffices to check that $\mathcal{H}$ does not collapse $E_{1}, E_{2}, E_{3}, \widetilde{C_{1}}, \ldots, \widetilde{C_{3}}$, or $\widetilde{C_{4}}$. 

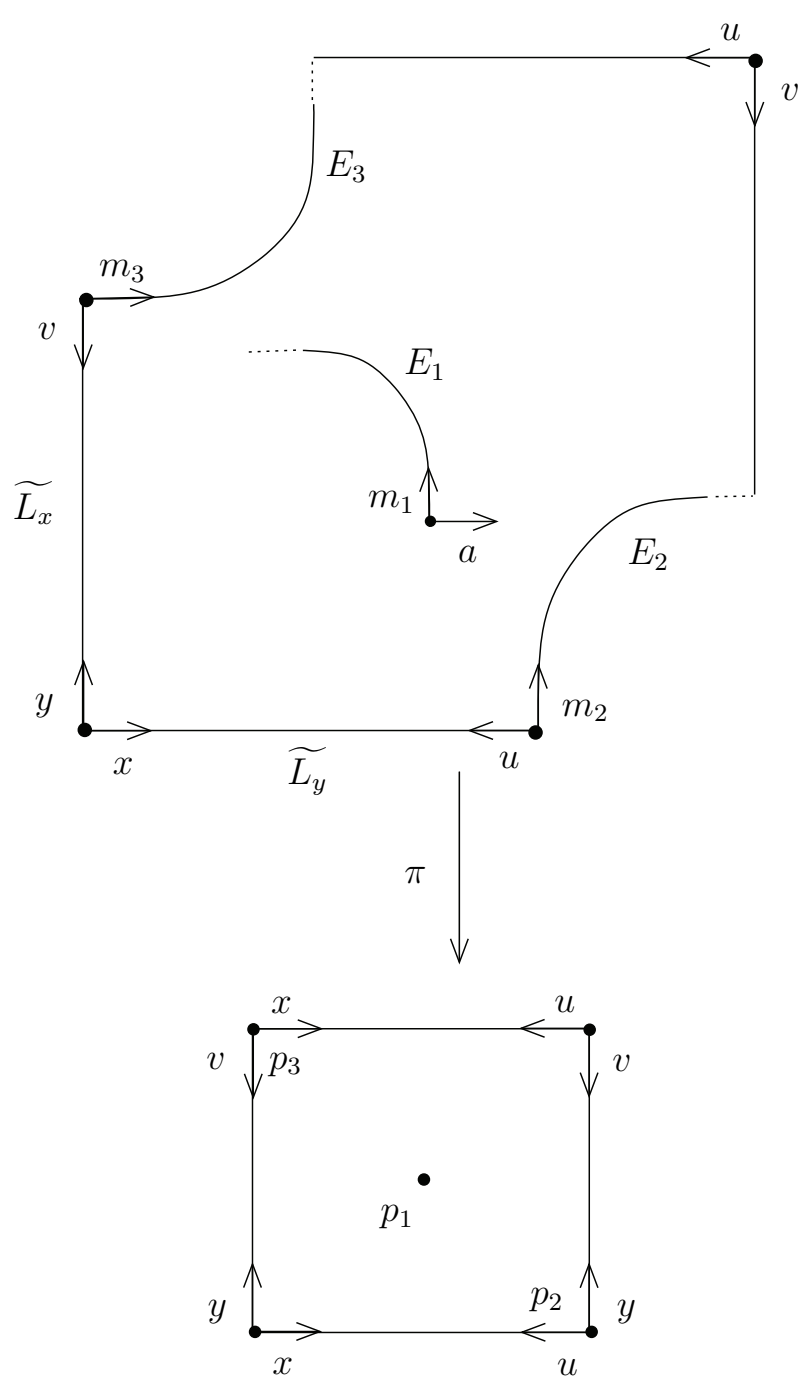

Figure 3 . On the bottom is $\mathbb{C P}^{1} \times \mathbb{C P}^{1}$, with the four sets of local coordinates labeled. On the top is $\mathcal{X}$, the blow-up of $\mathbb{C P}^{1} \times \mathbb{C P}^{1}$ at $p_{1}, p_{2}$, and $p_{3}$, with the respective exceptional divisors $E_{1}, E_{2}$, and $E_{3}$.

We will first check that $\mathcal{H}$ does not collapse the exceptional divisors $E_{1}, E_{2}$, and $E_{3}$. If we express $\mathcal{H}$ in using $\left(a, m_{1}\right)$ coordinates in the domain and $(x, y)$ in the codomain, we find

$x^{\prime}$

$=\frac{\left(a^{2} m_{1}^{2}+a m_{1}^{2}+4 a m_{1}+4 m_{1}+3\right)\left(a^{3} m_{1}^{2}+2 a^{2} m_{1}^{2}+2 a^{2} m_{1}+a m_{1}^{2}-2 a m_{1}+a-4 m_{1}-5\right)}{\left(a^{2} m_{1}^{2}+a m_{1}^{2}+6 a m_{1}+5 m_{1}+6\right)\left(a^{3} m_{1}^{2}+2 a^{2} m_{1}^{2}+2 a^{2} m_{1}+a m_{1}{ }^{2}-2 a m_{1}+a-5 m_{1}-4\right)}$

$y^{\prime}$

$=\frac{\left(a^{2} m_{1}+4 a m_{1}+a+3 m_{1}+4\right)\left(a^{3} m_{1}^{2}+2 a^{2} m_{1}^{2}+2 a^{2} m_{1}+a m_{1}^{2}-2 a m_{1}+a-5 m_{1}-4\right)}{\left(a^{2} m_{1}+6 a m_{1}+a+6 m_{1}+5\right)\left(a^{3} m_{1}^{2}+2 a^{2} m_{1}^{2}+2 a^{2} m_{1}+a m_{1}^{2}-2 a m_{1}+a-4 m_{1}-5\right)}$. 
The image of $E_{1}$ is obtained by setting $a=0$ :

$$
\left(x^{\prime}, y^{\prime}\right)=\left(\frac{\left(4 m_{1}+3\right)\left(4 m_{1}+5\right)}{\left(5 m_{1}+6\right)\left(5 m_{1}+4\right)}, \frac{\left(3 m_{1}+4\right)\left(5 m_{1}+4\right)}{\left(6 m_{1}+5\right)\left(4 m_{1}+5\right)}\right) .
$$

Since these functions are not constant functions of $m_{1}$, we conclude that $\mathcal{H}$ does not collapse $E_{1}$.

If we use the local coordinates $\left(u, m_{2}\right)$ in a neighborhood of generic points of $E_{2}$ and coordinates $(x, y)$ in the codomain, we find

$$
\begin{aligned}
x^{\prime} & =\frac{\left(m_{2}^{2} u+2 m_{2}-3\right)\left(m_{2}^{2} u-6 m_{2} u+6 u-1\right)}{\left(m_{2}^{2} u^{2}-m_{2} u^{2}+4 m_{2} u-5 u+1\right)\left(m_{2}^{2}-m_{2} u-6 m_{2}+6\right)}, \\
y^{\prime} & =\frac{u\left(2 m_{2} u+m_{2}-3 u\right)\left(m_{2}^{2}-m_{2} u-6 m_{2}+6\right)}{\left(m_{2} u^{2}+4 m_{2} u+m_{2}-5 u-1\right)\left(m_{2}^{2} u-6 m_{2} u+6 u-1\right)} .
\end{aligned}
$$

The image of $E_{2}$ is obtained by setting $u=0$ :

$$
\left(x^{\prime}, y^{\prime}\right)=\left(\frac{3-2 m_{2}}{m_{2}^{2}-6 m_{2}+6}, 0\right) \text {. }
$$

Again, since these functions are not constant functions of $m_{2}$, we conclude that $\mathcal{H}$ does not collapse $E_{2}$. The fact that $\mathcal{H}$ does not collapse $E_{3}$ follows by symmetry under $R$.

It remains to show that the curves $\widetilde{C}_{1}, \widetilde{C}_{2}, \widetilde{C}_{3}$, and $\widetilde{C}_{4}$ whose projections by $\pi$ were collapsed by $H$ are not collapsed by $\mathcal{H}$. We check $\widetilde{C}_{1}$ and $\widetilde{C}_{2}$ by expressing $\mathcal{H}$ using the local coordinates $(x, y)$ in the domain and $\left(a, m_{1}\right)$ in the codomain:

$$
\begin{aligned}
a^{\prime} & =-\frac{(2 x y+x+y-4)(x y-y-3)(x y-1)}{\left(x y^{2}+4 x y+x-y-5\right)\left(x^{2} y^{2}-6 x y-y+6\right)}, \\
m_{1}{ }^{\prime} & =\frac{\left(x^{2} y^{2}-6 x y-y+6\right)\left(x y^{2}+4 x y+x-y-5\right)(x y-x-3)}{(x y-y-3)\left(x^{2} y+4 x y-x+y-5\right)\left(x^{2} y^{2}-6 x y-x+6\right)} .
\end{aligned}
$$

Recall that $\widetilde{C}_{1}=\{x y-1=0\}$, and substituting $y=1 / x$ into the equation for $m_{1}{ }^{\prime}$, we have

$$
m_{1}^{\prime}=\frac{x(x+2)}{2 x+1},
$$

a nonconstant function. Thus, $m_{1}{ }^{\prime}$ varies while traversing $\widetilde{C}_{1}$, so $\mathcal{H}$ does not collapse $\widetilde{C}_{1}$. have

Similarly for $\widetilde{C}_{2}=\{2 x y+x+y-4=0\}$, substituting $y=\frac{4-x}{2 x+1}$ into $m_{1}{ }^{\prime}$, we

$$
m_{1}^{\prime}=\frac{9\left(x^{2}+x+1\right)}{\left(x^{2}+x+7\right)(2 x+1)} .
$$

This is also nonconstant, so $\mathcal{H}$ does not collapse $\widetilde{C}_{2}$. The defining equations for $\widetilde{C}_{1}$ and $\widetilde{C}_{2}$ appear in the numerator of $a^{\prime}$ so that they are both mapped by $\mathcal{H}$ to $E_{1}=\{a=0\}$. Since they are not collapsed by $\mathcal{H}$ and $E_{1}$ is irreducible, we conclude that $\widetilde{C}_{1}$ and $\widetilde{C}_{2}$ are mapped onto all of $E_{1}$. 
To check that $\widetilde{C}_{3}$ is mapped onto all of $E_{2}$, we use local coordinates $(x, y)$ in the domain and $\left(u, m_{2}\right)$ in the codomain:

$$
\begin{aligned}
u^{\prime} & =\frac{\left(x y^{2}+4 x y+x-y-5\right)\left(x^{2} y^{2}-6 x y-y+6\right)}{\left(x y^{2}+2 x y-3\right)\left(x^{2} y^{2}-6 x y-x+6\right)}, \\
m_{2}^{\prime} & =\frac{\left(x y^{2}+2 x y-3\right)\left(x^{2} y+2 x y-3\right)}{\left(x y^{2}+4 x y+x-y-5\right)\left(x^{2} y+4 x y-x+y-5\right)} .
\end{aligned}
$$

Since $(x, y)=(0,6)$ and $(x, y)=(1,6)$ are on $\widetilde{C}_{3}$ and are mapped by $\mathcal{H}$ to different points on $E_{2}$, we conclude that $\widetilde{C}_{3}$ is not collapsed by $\mathcal{H}$. Meanwhile, the defining equation for $\widetilde{C_{3}}$ is a factor of the numerator of the equation for $u^{\prime}$, so we conclude that $\mathcal{H}$ maps $\widetilde{C_{3}}$ onto all of $E_{2}=\{u=0\}$. By the symmetry of $\mathcal{H}$ under $R$, or very similar calculations, one can also show that $\widetilde{C}_{4}$ is mapped onto all of $E_{3}$.

We conclude that $\mathcal{H}$ does not collapse any curve. Therefore, Proposition 2.2 gives that $\mathcal{H}$ is algebraically stable.

\section{Pullback on cohomology and computation of $\lambda_{1}(H)$}

At this point we have completed all but the last step of the strategy for computing $\lambda_{1}(H)$ that was presented in Section 2.2. We have proved in Lemma 3.2 that $\mathcal{H}: \mathcal{X} \rightarrow \mathcal{X}$ is algebraically stable so we have

$$
\lambda_{1}(H)=\lambda_{1}(\mathcal{H})=\text { spectral radius }\left(\mathcal{H}^{*}: \mathrm{H}^{1,1}(\mathcal{X}) \rightarrow \mathrm{H}^{1,1}(\mathcal{X})\right) .
$$

It remains to use Lemma 2.1 to compute $\mathcal{H}^{*}: \mathrm{H}^{1,1}(\mathcal{X}) \rightarrow \mathrm{H}^{1,1}(\mathcal{X})$. The first step is to choose a good basis. It is well known that

$$
\mathrm{H}^{1,1}\left(\mathbb{C P}^{1} \times \mathbb{C P}^{1}\right) \cong \mathbb{Z}^{2}
$$

and is generated by $\left[L_{x}\right]=[\{X=0\}]$ and $\left[L_{y}\right]=[\{Y=0\}]$. Therefore, by [14, p. 474],

$$
\pi^{*}\left[L_{x}\right], \quad \pi^{*}\left[L_{y}\right], \quad\left[E_{1}\right], \quad\left[E_{2}\right], \quad \text { and } \quad\left[E_{3}\right]
$$

is an ordered basis for $\mathrm{H}^{1,1}(\mathcal{X})$, where $\pi^{*}\left[L_{x}\right]$ and $\pi^{*}\left[L_{y}\right]$ are the total transforms of $L_{x}$ and $L_{y}$ respectively.

Lemma 4.1. Let $C_{6}=\left\{x y^{2}+2 x y-3=0\right\}$ and $C_{7}=\left\{x^{2} y+2 x y-3=0\right\}$. We have

$$
\begin{aligned}
\mathcal{H}^{*}\left(\pi^{*}\left[L_{x}\right]\right) & =\left[\widetilde{C}_{6}\right]+\left[E_{3}\right]+\left[\widetilde{C}_{4}\right], \\
\mathcal{H}^{*}\left(\pi^{*}\left[L_{y}\right]\right) & =\left[\widetilde{C}_{7}\right]+\left[E_{2}\right]+\left[\widetilde{C}_{3}\right], \\
\mathcal{H}^{*}\left[E_{1}\right] & =\left[\widetilde{C}_{1}\right]+\left[\widetilde{C}_{2}\right], \\
\mathcal{H}^{*}\left[E_{2}\right] & =\left[\widetilde{C}_{3}\right], \quad \text { and } \\
\mathcal{H}^{*}\left[E_{3}\right] & =\left[\widetilde{C_{4}}\right] .
\end{aligned}
$$

Proof. We'll start by computing $\mathcal{H}^{*}\left[E_{i}\right]$ for each $1 \leq i \leq 3$. If we express $\mathcal{H}$ in local coordinates $(x, y)$ in the domain and $\left(a, m_{1}\right)$ in the codomain, as in (3.5), $E_{1}$ is locally $\{a=0\}$. We see that the defining equations for both $C_{1}$ and $C_{2}$ appear exactly once in the numerator of the expression for $a^{\prime}$, so that $\left[\widetilde{C}_{1}\right]$ and $\left[\widetilde{C}_{2}\right]$ are each assigned multiplicity one in $\mathcal{H}^{*}\left[E_{1}\right]$. (Had the defining equation of $C_{1}$, for 
example, appeared with an exponent larger than one in the expression for $a^{\prime}$, the coefficient on homology would correspond to that exponent.) The only additional term in the product is $x y-x-3$. However, this also appears in the denominator of the expression for $m_{1}{ }^{\prime}$, so the curve $\{x y-x-3=0\}$ is mapped to a line at infinity. This proves (4.5).

The only curve mapping by $\mathcal{H}$ to $E_{2}$ is $\widetilde{C_{3}}$. A calculation in local coordinates like above shows that $\left[\widetilde{C_{3}}\right]$ occurs with multiplicity one in $\mathcal{H}^{*}\left[E_{2}\right]$, proving (4.6). Again, we get (4.7) by symmetry.

Lastly, we compute $\mathcal{H}^{*}\left(\pi^{*}\left[L_{y}\right]\right)$ and then obtain $\mathcal{H}^{*}\left(\pi^{*}\left[L_{x}\right]\right)$ by symmetry under $R$. Note that

$$
\mathcal{H}^{*}\left(\pi^{*}\left[L_{y}\right]\right)=\mathcal{H}^{*}\left(\left[\widetilde{L_{y}}\right]+\left[E_{2}\right]\right)=\mathcal{H}^{*}\left[\widetilde{L_{y}}\right]+\left[\widetilde{C}_{3}\right]
$$

where $\widetilde{L_{y}}$ is the proper transform of $L_{y}$. It remains to compute $\mathcal{H}^{*}\left[\widetilde{L_{y}}\right]$. One sees from (1.1) that the only curves mapped by $H$ to $L_{y}$ are $C_{3}$ and $C_{7}$. After performing the blowups, we saw in Lemma 3.2 that $\mathcal{H}$ maps $\widetilde{C_{3}}$ onto $E_{2}$. Therefore, $\widetilde{C_{3}}$ does not contribute to $\mathcal{H}^{*}\left[\widetilde{L_{y}}\right]$. We also saw in (3.4) that $\mathcal{H}$ maps $E_{2}$ onto $\widetilde{L_{y}}$. Thus, $\mathcal{H}^{*}\left[\widetilde{L_{y}}\right]$ is a sum of $\left[\widetilde{C_{7}}\right]$ and $\left[E_{2}\right]$ with suitable multiplicities. The defining equation for $C_{7}$ occurs with multiplicity one in the equation for $y^{\prime}$ in (1.1). Meanwhile, $E_{2}$ is $\{u=0\}$ in the $\left(u, m_{2}\right)$ coordinates. Since $u$ occurs with multiplicity one in the equation for $y^{\prime}$ in (3.3), we see that $\left[E_{2}\right]$ is also assigned multiplicity one in $\mathcal{H}^{*}\left[\widetilde{L_{y}}\right]$. This proves (4.4), and equation (4.3) follows immediately under the symmetry $R$.

Remark 4.2. Some caution is required when computing $\mathcal{H}^{*}\left(\pi^{*}\left[L_{y}\right]\right)$. Indeed,

$$
\pi^{*} \circ H^{*} \neq(\mathcal{H} \circ \pi)^{*}=(\pi \circ H)^{*}=\mathcal{H}^{*} \circ \pi^{*}
$$

where the first inequality comes from the fact that $\pi$ collapses curves into the indeterminacy points of $H$ and the Diller-Favre criterion (Proposition 2.2), the middle equality comes from commutative diagram (3.2), and the last equality comes from the fact that $\mathcal{H}$ does not collapse any curves (Lemma 3.2) and again the DillerFavre criterion.

More specifically, one can check that $H^{*}\left[L_{y}\right]=\left[C_{3}\right]+\left[C_{7}\right]$, so

$$
\begin{aligned}
\pi^{*}\left(H^{*}\left[L_{y}\right]\right) & =\pi^{*}\left(\left[C_{3}\right]+\left[C_{7}\right]\right)=\left[\widetilde{C}_{3}\right]+\left[\widetilde{C}_{7}\right]+2\left[E_{1}\right]+2\left[E_{2}\right]+2\left[E_{3}\right] \\
& \neq \mathcal{H}^{*}\left(\pi^{*}\left[L_{y}\right]\right) .
\end{aligned}
$$

We now need to re-express each of the cohomology classes on the right hand sides of (4.3 4.7) in terms of the ordered basis (4.2). We first express the cohomology 
classes $\left[C_{1}\right], \ldots,\left[C_{4}\right],\left[C_{6}\right]$ and $\left[C_{7}\right]$ in the basis $\left[L_{x}\right]$ and $\left[L_{y}\right]$ for $\mathrm{H}^{1,1}\left(\mathbb{C P}^{1} \times \mathbb{C P}^{1}\right)$ :

Lemma 4.3. In $\mathrm{H}^{1,1}\left(\mathbb{C P}^{1} \times \mathbb{C P}^{1}\right)$ we have

$$
\begin{aligned}
& {\left[C_{1}\right]=\left[L_{x}\right]+\left[L_{y}\right],} \\
& {\left[C_{2}\right]=\left[L_{x}\right]+\left[L_{y}\right],} \\
& {\left[C_{3}\right]=2\left[L_{x}\right]+2\left[L_{y}\right],} \\
& {\left[C_{4}\right]=2\left[L_{x}\right]+2\left[L_{y}\right],} \\
& {\left[C_{6}\right]=\left[L_{x}\right]+2\left[L_{y}\right], \quad \text { and }} \\
& {\left[C_{7}\right]=2\left[L_{x}\right]+\left[L_{y}\right] .}
\end{aligned}
$$

Proof. Equations (4.8) - (4.13) follow from the well-known fact that if a curve $C \subset \mathbb{C P}^{1} \times \mathbb{C P}^{1}$ is defined in standard affine coordinates by a polynomial $p(x, y)=0$, then

$$
[C]=d_{x}\left[L_{x}\right]+d_{y}\left[L_{y}\right]
$$

where $d_{x}$ is the degree of $p$ with respect to $x$, and $d_{y}$ is the degree of $p$ with respect to $y$.

We now lift the results of Lemma 4.3 to the blown-up space $\mathcal{X}$ :

Lemma 4.4. In $\mathrm{H}^{1,1}(\mathcal{X})$ we have

$$
\begin{aligned}
& {\left[\widetilde{C_{1}}\right]=\pi^{*}\left[L_{x}\right]+\pi^{*}\left[L_{y}\right]-\left[E_{1}\right]-\left[E_{2}\right]-\left[E_{3}\right],} \\
& {\left[\widetilde{C_{2}}\right]=\pi^{*}\left[L_{x}\right]+\pi^{*}\left[L_{y}\right]-\left[E_{1}\right],} \\
& {\left[\widetilde{C_{3}}\right]=2 \pi^{*}\left[L_{x}\right]+2 \pi^{*}\left[L_{y}\right]-\left[E_{1}\right]-2\left[E_{2}\right]-\left[E_{3}\right],} \\
& {\left[\widetilde{C_{4}}\right]=2 \pi^{*}\left[L_{x}\right]+2 \pi^{*}\left[L_{y}\right]-\left[E_{1}\right]-\left[E_{2}\right]-2\left[E_{3}\right],} \\
& {\left[\widetilde{C}_{6}\right]=\pi^{*}\left[L_{x}\right]+2 \pi^{*}\left[L_{y}\right]-\left[E_{1}\right]-\left[E_{2}\right]-\left[E_{3}\right], \quad \text { and }} \\
& {\left[\widetilde{C}_{7}\right]=2 \pi^{*}\left[L_{x}\right]+\pi^{*}\left[L_{y}\right]-\left[E_{1}\right]-\left[E_{2}\right]-\left[E_{3}\right] .}
\end{aligned}
$$

Proof. Since $C_{1}$ has multiplicity one at $p_{1}, p_{2}$, and $p_{3}$, and $\left[C_{1}\right]=\left[L_{x}\right]+\left[L_{y}\right]$ by Lemma 4.3. we can pull back both sides of the equation by $\pi$ to see that

$$
\left[\widetilde{C_{1}}\right]+\left[E_{1}\right]+\left[E_{2}\right]+\left[E_{3}\right]=\pi^{*}\left[L_{x}\right]+\pi^{*}\left[L_{y}\right] .
$$

Equation (4.14) follows. Equations (4.15,4.19) follow by similar calculations. The only subtlety is computing the local multiplicities, which we summarize here:

\begin{tabular}{|c||c|c|c|}
\hline Curve & mult. at $p_{1}$ & mult. at $p_{2}$ & mult. at $p_{3}$ \\
\hline \hline$C_{2}$ & 1 & 0 & 0 \\
\hline$C_{3}$ & 1 & 2 & 1 \\
\hline$C_{4}$ & 1 & 1 & 2 \\
\hline$C_{6}$ & 1 & 1 & 1 \\
\hline$C_{7}$ & 1 & 1 & 1 \\
\hline
\end{tabular}


Lemmas 4.1 and 4.4 make it easy to express $\mathcal{H}^{*}: \mathrm{H}^{1,1}(\mathcal{X}) \rightarrow \mathrm{H}^{1,1}(\mathcal{X})$ in the ordered basis (4.2). For example,

$$
\mathcal{H}^{*}\left[\widetilde{L_{x}}\right]=\left[\widetilde{C}_{6}\right]+\left[E_{3}\right]+\left[\widetilde{C}_{4}\right]=3 \pi^{*}\left[L_{x}\right]+4 \pi^{*}\left[L_{y}\right]-2\left[E_{1}\right]-2\left[E_{2}\right]-2\left[E_{3}\right] .
$$

Computing all of the others, we find

$$
\mathcal{H}^{*}=\left[\begin{array}{ccccc}
3 & 4 & 2 & 2 & 2 \\
4 & 3 & 2 & 2 & 2 \\
-2 & -2 & -2 & -1 & -1 \\
-2 & -2 & -1 & -2 & -1 \\
-2 & -2 & -1 & -1 & -2
\end{array}\right] .
$$

This matrix has characteristic polynomial $-(4-\lambda)(1+\lambda)^{4}$. The spectral radius is the largest root, which is 4 . Using (4.1) we have that $\lambda_{1}(H)=\lambda_{1}(\mathcal{H})=4$.

$\square$ (Theorem 1.1).

Remark 4.5. There seems to be no convention on how to express a pullback on cohomology in matrix notation. We have written (4.21) so that $\mathcal{H}^{*}$ acts on column vectors. However there are many other papers where the transpose is used.

\section{CONCluding QUeSTiOns AND REMARKS}

Computing the dynamical degrees of $H$ and gleaning the immediate dynamical consequences is really just the first step towards an understanding of it as a complex dynamical system. There are many interesting avenues for further study and connections with other areas. Among them, we list the following two:

5.1. Complex proof of theorem on convex classes. One of the key theorems in Schwartz's monograph [19] is that (the projective equivalence class) of any convex pentagon has orbit under $H$ converging to (the projective equivalence class) of the regular pentagon. He gives a computer assisted proof. Can it be done using techniques from complex dynamics? Can it be generalized to arbitrary $n$-gons? A solution would probably require a suitable complex notion of "convex".

5.2. Polarized dynamical system. The eigenvector of $\mathcal{H}^{*}$ corresponding to $\lambda=4$ is

$$
-K_{\mathcal{X}}=2 \pi^{*}\left[L_{x}\right]+2 \pi^{*}\left[L_{y}\right]-\left[E_{1}\right]-\left[E_{2}\right]-\left[E_{3}\right] .
$$

The referee pointed out the interesting fact that this is the anticanonical class of $\mathcal{X}$ (i.e. minus the canonical class of $X$ ); see [14, p. 146, p. 187].

Recall that one can obtain $\mathbb{C P}^{1} \times \mathbb{C P}^{1}$ by blowing up $\mathbb{C P}^{2}$ at two points and then blowing down the proper transform of the line between them. Using this fact, one can recognize that our surface $\mathcal{X}$ is isomorphic to the blow up of $\mathbb{C P}^{2}$ at $[0: 0: 1],[0: 1: 0],[1: 0: 0]$ and $[1: 1: 1]$. Such a surface is a del Pezzo surface [1, 12] and thus $-K_{X}$ is ample. Because we have

$$
\mathcal{H}^{*}\left(-K_{\mathcal{X}}\right)=4\left(-K_{\mathcal{X}}\right)
$$

with $-K_{\mathcal{X}}$ ample, $\mathcal{H}: \mathcal{X} \rightarrow \mathcal{X}$ is called a polarized dynamical system [16,21. Polarized dynamical systems have been primarily of interest in arithmetic and algebraic dynamics and they are usually studied for endomorphisms (i.e. rational mappings 
without indeterminate points). However, we mention the polarization for $\mathcal{H}$ in case there is a connection with arithmetic dynamics, despite the indeterminate points of $\mathcal{H}$, or in case it can be used for some other purpose.

\section{ACKNOWLEDGMENTS}

We thank the referee for several helpful comments and Joseph Silverman for his comments on polarized dynamical systems. The second author was supported by NSF grant DMS-1348589.

\section{REFERENCES}

[1] Wikipedia page on del pezzo surfaces https://en.wikipedia.org/wiki/ Del_Pezzo_surface.

[2] Eric Bedford, The dynamical degrees of a mapping, Proceedings of the Workshop Future Directions in Difference Equations, Colecc. Congr., vol. 69, Univ. Vigo, Serv. Publ., Vigo, 2011, pp. 3-13. MR2905566

[3] Jean-Yves Briend and Julien Duval, Exposants de Liapounoff et distribution des points périodiques d'un endomorphisme de $\mathbf{C P}^{k}$ (French), Acta Math. 182 (1999), no. 2, 143-157, DOI 10.1007/BF02392572. MR:1710180

[4] Jean-Yves Briend and Julien Duval, Deux caractérisations de la mesure d'équilibre d'un endomorphisme de $P^{k}(\mathbb{C})$, Publications Mathématiques de l'IHÉS 93 (2001), 145-159.

[5] J. Diller and C. Favre, Dynamics of bimeromorphic maps of surfaces, Amer. J. Math. 123 (2001), no. 6, 1135-1169. MR1867314

[6] Jeffrey Diller, Romain Dujardin, and Vincent Guedj, Dynamics of meromorphic maps with small topological degree I: from cohomology to currents, Indiana Univ. Math. J. 59 (2010), no. 2, 521-561, DOI 10.1512/iumj.2010.59.4023. MR2648077

[7] Tien-Cuong Dinh and Viêt-Anh Nguyên, Comparison of dynamical degrees for semiconjugate meromorphic maps, Comment. Math. Helv. 86 (2011), no. 4, 817-840, DOI 10.4171/CMH/241. MR2851870

[8] Tien-Cuong Dinh, Viêt-Anh Nguyên, and Tuyen Trung Truong, On the dynamical degrees of meromorphic maps preserving a fibration, Commun. Contemp. Math. 14 (2012), no. 6, 1250042, 18, DOI 10.1142/S0219199712500423. MR2989646

[9] Tien-Cuong Dinh, Viêt-Anh Nguyên, and Tuyen Trung Truong, Equidistribution for meromorphic maps with dominant topological degree, Indiana Univ. Math. J. 64 (2015), no. 6, 1805-1828, DOI 10.1512/iumj.2015.64.5674. MR.3436236

[10] Tien-Cuong Dinh and Nessim Sibony, Regularization of currents and entropy (English, with English and French summaries), Ann. Sci. École Norm. Sup. (4) 37 (2004), no. 6, 959-971, DOI 10.1016/j.ansens.2004.09.002. MR2119243

[11] Tien-Cuong Dinh and Nessim Sibony, Une borne supérieure pour l'entropie topologique d'une application rationnelle, Annals of mathematics (2005), 1637-1644.

[12] Igor V. Dolgachev, Classical algebraic geometry, Cambridge University Press, Cambridge, 2012. A modern view. MR2964027

[13] Shmuel Friedland, Entropy of polynomial and rational maps, Ann. of Math. (2) 133 (1991), no. 2, 359-368, DOI 10.2307/2944341. MR.1097242

[14] Phillip Griffiths and Joseph Harris, Principles of algebraic geometry, Wiley-Interscience [John Wiley \& Sons], New York, 1978. Pure and Applied Mathematics. MR507725

[15] Vincent Guedj, Ergodic properties of rational mappings with large topological degree, Ann. of Math. (2) 161 (2005), no. 3, 1589-1607, DOI 10.4007/annals.2005.161.1589. MR2179389

[16] Shu Kawaguchi, Introduction to algebraic and arithmetic dynamics - a survey, Algebraic number theory and related topics 2009, RIMS Kôkyûroku Bessatsu, B25, Res. Inst. Math. Sci. (RIMS), Kyoto, 2011, pp. 103-123. MR2868573

[17] Roland K. W. Roeder, The action on cohomology by compositions of rational maps, Math. Res. Lett. 22 (2015), no. 2, 605-632, DOI 10.4310/MRL.2015.v22.n2.a13. MR3342248

[18] Alexander Russakovskii and Bernard Shiffman, Value distribution for sequences of rational mappings and complex dynamics, Indiana Univ. Math. J. 46 (1997), no. 3, 897-932, DOI 10.1512/iumj.1997.46.1441. MR.1488341 
[19] Rich E. Schwartz, The projective heat map, AMS Mathematical Surveys and Monographs, Vol. 219, American Mathematical Society, Providence, 2017. ISBN: 978-1-4704-3514-1.

[20] Igor R. Shafarevich, Basic algebraic geometry. 1, 2nd ed., Springer-Verlag, Berlin, 1994. Varieties in projective space; Translated from the 1988 Russian edition and with notes by Miles Reid. MR 1328833

[21] Shou-Wu Zhang, Distributions in algebraic dynamics, Surveys in differential geometry. Vol. X, Surv. Differ. Geom., vol. 10, Int. Press, Somerville, MA, 2006, pp. 381-430, DOI 10.4310/SDG.2005.v10.n1.a9. MR2408228

Department of Mathematics \& Actuarial Science, Butler University, Jordan Hall, Room 270, 4600 Sunset Avenue, Indianapolis, Indiana 46208

E-mail address: skaschne@butler.edu

Department of Mathematical Sciences, IUPUi, LD Building, Room 224Q, 402 North BlackFord Street, Indianapolis, Indiana 46202-3267

E-mail address: rroeder@math.iupui.edu 